\title{
Oncologie cellulaire et moléculaire
}

\section{Hugues de Thé}

\section{OpenEdition}

Journals

Édition électronique

URL : https://journals.openedition.org/annuaire-cdf/15566

DOI : 10.4000/annuaire-cdf.15566

ISBN : 978-2-7226-0572-5

ISSN : 2109-9227

Éditeur

Collège de France

Édition imprimée

Date de publication : 30 décembre 2020

Pagination : 131-138

ISBN : 978-2-7226-0516-9

ISSN : 0069-5580

Référence électronique

Hugues de Thé, "Oncologie cellulaire et moléculaire », L'annuaire du Collège de France [En ligne], 118| 2020, mis en ligne le 01 avril 2021, consulté le 22 août 2022. URL : http://journals.openedition.org/ annuaire-cdf/15566 ; DOI : https://doi.org/10.4000/annuaire-cdf.15566 


\title{
ONCOLOGIE CELLULAIRE ET MOLÉCULAIRE
}

\author{
Hugues DE THÉ \\ Membre de l'Institut (Académie des sciences), \\ professeur au Collège de France
}

Mots-clés : oncologie, cancer, hématopoïèse

La série de cours «Facteurs de transcription et différenciation cellulaire » est disponible en vidéo sur le site internet du Collège de France (https://www.college-defrance.fr/site/hugues-de-the/course-2017-2018.htm), ainsi que le colloque «Master transcriptional regulators and cancer » (https://www.college-de-france.fr/site/huguesde-the/symposium-2017-2018.htm).

\section{ENSEIGNEMENT}

\section{INTRODUCTION}

La régulation de l'expression des gènes est un facteur essentiel du développement embryonnaire précoce. C'est également une partie intégrante de la transformation cancéreuse. Il n'est donc pas surprenant que de nombreux gènes maîtres du développement entretiennent des liens étroits avec les cancers. Par exemple, dans le domaine des leucémies, les plus importants régulateurs de la différenciation initiale des cellules souches hématopoïétiques ont été découverts à l'occasion de leur implication dans des translocations chromosomiques associées à des leucémies. Ce sont ces liaisons dangereuses que le cours de cette année se proposait de revisiter.

Quelques rappels sur les origines génétiques du cancer ont été présentés, en particulier la notion de mutation driver, directement impliquée dans le développement de ces pathologies. Dans le cas des leucémies, le nombre de ces drivers peut être parfois très limité, un à deux. Si le catalogue des mutations associées aux différentes formes de cancer commence à être bien connu, les fonctions cellulaires exactes de ces mutants dans la cellule d'origine restent souvent très mal comprises. Les protéines altérées dans des cancers sont souvent situées à des nœuds de signalisations et contrôlent de ce fait de multiples voies (prolifération, métabolisme, apoptose, 
sénescence, différenciation, migration...). Dans ce contexte, le rôle exact de chacune des pertes ou de chacun des gains de fonction associés à une mutation donnée, reste souvent flou. C'est l'enjeu de la post-génomique que d'explorer ces différentes facettes fonctionnelles, étapes essentielles mais souvent négligées, à la mise au point de thérapeutiques ciblées.

L'étude des leucémies a été facilitée par la bonne connaissance des différentes étapes de la différenciation hématopoïétique normale. La transcription des gènes fait l'objet de multiples régulations dont les grands principes ont été rappelés en s'appuyant sur la récente revue de J.E. Bradner dans Cell. Une emphase particulière a été portée sur les translocations chromosomiques et les anomalies des promoteurs des gènes maîtres de la transformation. Les liens entre les mutations du complexe cohésine, élément clé de l'architecture de la chromatine, et la transformation des progéniteurs hématopoïétiques ont été présentés. L'importance de l'analyse des effets de ces mutations dans le bon type cellulaire a été soulignée. Technologiquement, l'analyse de mutants ponctuels in vivo, par les approches de knock-in dans des modèles de souris, constitue aujourd'hui l'approche de choix. Les techniques expérimentales (inactivation génique complète, inactivation tissu-spécifique, inactivation tissu-spécifique inductible, excision des éléments régulateurs, suivi des progéniteurs et analyse de hiérarchie cellulaire par lineage tracing, expression inductible d'un gène spécifique) ont été rapidement exposées.

\section{COURS - FACTEURS DE TRANSCRIPTION ET DIFFÉRENCIATION CELLULAIRE}

Le premier exemple que nous avons choisi pour illustrer le concept de gêne maître est celui de l'œil. Le gène Pax6 est indispensable à sa formation dans un très vaste éventail d'espèces. Inversement, l'expression ectopique de Pax6 in vivo va induire la formation d'yeux ailleurs dans l'organisme, comme l'a démontré W. Gehring. Cet exemple extrême de trans-différenciation a été maintenant étendu à un grand nombre de couples gènes maîtres-types cellulaires comme MyoD/muscle, CEBPA/ macrophage, Pax5/lymphocyte B, Oct4/progéniteur hématopoḯtiques....

Nous avons choisi d'approfondir deux exemples de gènes maîtres de la différenciation des cellules sanguines : CEBPA et RUNX1. RUNX1 est un gène très ancien dont un homologue existe chez l'amibe, où il régule la taille cellulaire. Chez le nématode C. elegans, un homologue de Runx1 régule la division asymétrique des cellules souches. Des duplications tardives ont permis la diversification du gène ancestral en trois gènes Runx chez les mammifères. Runx1 joue un rôle clé dans l'hématopoïèse, Runx 2 dans la chondrogénèse et Runx 3 dans la neurogénèse. La séquence du domaine de liaison à l'ADN est largement conservée parmi les membres de la famille. Il hétérodimérise avec un partenaire unique $\mathrm{CBF} \beta$ et se lie à des éléments de réponse canoniques. Des analyses génétiques chez $C$. elegans ont démontré que la perte de Runt ou de l'homologue de CBF $\beta$ ont des effets très similaires. Outre le domaine de liaison à l'ADN, les membres de la famille Runt/Runx ont des domaines de régulation (activation ou répression) de la transcription qui fixent de nombreux régulateurs, en particulier épigénétiques. Certains de ces régulateurs sont des facteurs de transcription (Myb, Ets, SMAD ...) qui contribuent à la fixation des complexes Runx/CBF $\beta$ sur des promoteurs spécifiques. Ainsi, ces partenaires contribuent à l'extrême diversité des fonctions biologiques associées à la famille. Au niveau moléculaire, l'expression de gènes Runx est assurée par deux promoteurs, $\mathrm{P} 1$ et $\mathrm{P} 2$, fortement conservés dans 
l'évolution. P2 est le promoteur ancestral doté d'une activité constitutive. P1 est induit par Runx1, mais inhibé par Runx2. Il y a donc un réseau de signalisation autorégulé et toute anomalie d'un membre de la famille va retentir sur les autres. Les protéines traduites à partir des ARNm exprimés depuis $\mathrm{P} 1$ ou $\mathrm{P} 2$ présentent quelques acides aminés N-terminaux de différence. Ceux-ci ont récemment été impliqués dans la formation des plaquettes sanguines.

De manière inattendue, les effets biologiques des Runx peuvent parfois être opposés dans des types cellulaires différents. Ainsi, les surexpressions de Runx1, mais aussi Runx 2 ou 3 vont promouvoir la transformation cancéreuse. Mais inversement, l'excision de Runx1 à la naissance va aussi favoriser la lymphomatogénèse induite par une infection rétrovirale ! De même, l'excision de Runx3 dans le poumon favorise la transformation par K-RasG12D. Ainsi, suivant le contexte cellulaire, la surexpression ou la sous-expression peuvent l'une et l'autre favoriser la transformation cancéreuse. Fonctionnellement, les cibles de Runx1 sont la différenciation hématopoïétique primitive, la biosynthèse des ribosomes et la prolifération cellulaire. Cette dernière est contrôlée à travers les signalisations p53 et TGF $\beta$. L'excision de Runx1 est létale, du fait de l'absence complète des cellules sanguines. L'expression in vivo de protéines rapporteuses (GFP ou $\beta$-galactosidase) a permis de spécifier les territoires d'expression de Runx1 dès les premiers stades de l'embryogénèse. Runx 1 permet de tracer l'origine des premières cellules sanguines, depuis le sac vitellin, jusqu'à l'aorte de l'embryon. Des études élégantes ont permis de visualiser la transition entre l'endothélium vasculaire et les premières cellules souches hématopoïétiques, transition dans laquelle Runx1 joue un rôle essentiel. Comme chez $C$. elegans, l'inactivation du partenaire CBF $\beta$ a les mêmes effets que celle de Runx1, démontrant ainsi le rôle clé de l'hétérodimère dans la spéciation des cellules hématopoḯtiques. De manière remarquable, l'inactivation de Runx1 à la naissance n'a que des effets modestes, ne conduisant qu'à une perte lente des cellules souches hématopoïétiques du fait d'une augmentation de leur différenciation spontanée. Ainsi, cette protéine est indispensable à la formation des progéniteurs, puis, dans une moindre mesure, à leur maintien. L'excision de P2 mime la perte totale du gène, démontrant son rôle essentiel dans l'amorçage de ce réseau de signalisation. Néanmoins, s'il est indispensable, le promoteur P2 n'est pas suffisant pour conférer le patron si spécifique d'expression spatio-temporelle de ce gène dans l'embryon précoce. Ceci est assuré par un « augmenteur » récemment identifié, situé à distance du gène. Les fonctions de Runx 1 dans le développement sont assurées par l'activation de nombreux gènes cibles. L'un des principaux est PU-1, un autre gène maître de l'hématopoïèse, qui se lie à Runx1 pour former des complexes activateurs ou répresseurs suivant les stades de la différenciation.

L'une des raisons ayant justifié l'étude très poussée de la famille Runx est son implication étroite dans plusieurs pathologies humaines. Runx1 est directement impliqué dans de nombreux type de leucémies, les mutations de Runx2 dans des syndromes développementaux touchant les os ou cartilages, et la méthylation de Runx3 dans des tumeurs épithéliales, en particulier de l'estomac. Les liens entre anomalies de Runx1 et prolifération des lignées sanguines sont probablement les mieux compris, même s'il reste encore une part de mystère. Runx1 et son partenaire $\mathrm{CBF} \beta$ sont les cibles de translocations récurrentes associées à des leucémies aiguës myéloïdes ou lymphoïdes. Les modèles actuels de physiopathologie font appel à des effets dominants-négatifs des produits de fusion dérivés des translocations. La fusion Runx1/Eto est associée aux leucémies aiguës myéloïdes de l'enfant. Cette fusion 
réprime l'expression de deux gènes maîtres d'aval, PU-1 et CEBPA (cf. infra) grâce à une autodimérisation par sa partie Eto et au recrutement de corépresseurs. Les premières souris knock-in pour la fusion ont présenté un phénotype de létalité embryonnaire extrêmement proche de celui de l'inactivation germinale de Runx1, démontrant son activité dominante négative. Toutefois, il existe une petite population de pro-géniteurs capables d'être propagés à long terme, population qui n'est jamais observée avec l'inactivation simple de Runx1. Ainsi, la fusion permet une certaine forme d'autorenouvellement des progéniteurs. Cette dernière constitue probablement le préambule à la transformation cellulaire. L'exploration des fusions CBF $\beta / M y h 11$, associées à une autre forme morphologique de leucémies myéloïdes, a donné des résultats très similaires à ceux obtenus avec les fusions Runx 1/Eto.

Les fusions Tel/Runx1 sont, quant à elles, associées à des leucémies aiguës lymphoïdes pré-B. Là encore, ces fusions sont capables de former des dimères et de recruter des corépresseurs. L'allèle normal de Tel est souvent secondairement perdu. Cette pathologie de l'enfant a permis de remarquables études de concordance entre jumeaux. Celle-ci est d'environ $5 \%$ chez les jumeaux monochoriaux. S'il y a concordance, le point de translocation chromosomique des deux leucémies est identique, démontrant que la translocation a été acquise in utero et que le clone primordial a donc circulé d'un jumeau à l'autre. En revanche, les événements secondaires, comme les pertes de Tel, sont différentes entre les leucémies observées chez chacun des jumeaux. Cette translocation est retrouvée chez environ $1 \%$ des nouveau-nés. Cela suggère que l'événement de translocation est assez courant et est capable d'induire une prolifération transitoire des pro-géniteurs hématopoïétiques. Néanmoins, ce clone est le plus souvent perdu, peut-être du fait de la sénescence oncogénique, expliquant la concordance de seulement $5 \%$ chez les jumeaux. Enfin, des études menées sur les rechutes ont établi que celles-ci sont souvent des leucémies distinctes de la première (par exemple par le patron de perte de Tel), mais toujours dérivées du clone primordial. Contrairement à la leucémie, ce clone primordial résiste donc à la chimiothérapie (voir travaux de recherche). Ainsi, ces études ont apporté un éclairage précieux sur les étapes évolutives de la leucémogenèse chez l'homme.

Il reste néanmoins un certain nombre de paradoxes à éclaircir dans la leucémogenèse associée aux anomalies de Runx1. Le maintien d'un l'allèle de Runx 1 est essentiel, ce qui peut paraître en contradiction avec le modèle dominant négatif, même s'il existe, grâce à la dimérisation, un gain de cible. Enfin, l'exploration des cibles de CBF $\beta /$ Myh11 a plutôt mis en évidence des marques d'activation ! Il existe des mutations mono- ou bi-alléliques de Runx1 dans de très nombreuses autres formes de leucémies ou de syndromes pré-leucémiques. Il a été proposé que leurs effets oncogéniques soient liés à une haplo-insuffisance. Néanmoins, des gains de fonction (à l'image des mutants de P53 analysés dans un cours précédent) ne peuvent pas être totalement éliminés. Ces mutations peuvent être germinales et entrer dans le cadre de syndromes de prédisposition. Environ la moitié des sujets porteurs de ces mutations germinales de Runx1 vont un jour développer une leucémie, mais sans perte de l'allèle normal, à la différence des modèles classiques où c'est la perte du second allèle qui initie la maladie. On ne comprend pas à ce jour quelles sont les gènes cibles essentiels à l'expansion des pro-géniteurs porteurs de ces translocations. Des travaux menés sur l'homologue de Runx1 chez la mouche ont montré que certaines fonctions essentielles ne nécessitent pas le site de liaison à l'ADN! Toutefois, la connaissance, encore 
imparfaite, de cette voie de signalisation a conduit au développement de stratégies thérapeutiques innovantes, par exemple en ciblant le domaine de dimérisation des fusions CBF $\beta /$ Myh11.

Le dernier cours a présenté un autre gène maître de la différenciation hématopoïétique, CEBPA, un membre d'une famille de facteur de transcription ayant un domaine de liaison à l'ADN de type « leucine zipper ». Il s'agit d'un facteur dit « pionnier», capable de se fixer à une chromatine condensée, de l'ouvrir et de déplacer les nucléosomes par recrutement du complexe SWI/SNIF. Cette ouverture permet la fixation secondaire de facteurs instructifs de lignage. Ces propriétés expliquent qu'il soit indispensable à plusieurs voies de différenciation et qu'il favorise la reprogrammation cellulaire. Dans la différenciation hématopoïétique normale, l'expression de CEBPA est indispensable pour la différenciation granulomonocytaire et l'inhibition de la différenciation érythroïde. Ainsi, l'inactivation germinale arrête la différenciation myéloïde au stade de pro-géniteur. À l'inverse, l'expression forcée dans des lymphocytes B permet une réversion phénotypique vers les granuleux, illustrant un exemple frappant de transdifférenciation.

L'expression de CEBPA est directement induite par le complexe Runx1/PU-1, mais CEBPA induit également PU-1, rétrocontrôle positif qui facilite l'irréversibilité de la différenciation normale. Si CEPBA est exprimé dans de nombreux tissus, les séquences régulatrices de type «augmenteur» dirigeant son expression dans les lignées sanguines, ont été localisées à - $42 \mathrm{~kb}$ du promoteur proximal. L'excision de cet élément régulateur abroge l'expression dans les lignées sanguines, mais pas dans le foie ou le tissu adipeux. Deux modifications post-traductionnelles essentielles à l'activité de CEBPA ont été identifiées. L'acétylation et la conjugaison à SUMO1 diminuent fortement son activité et elles ont été impliquées dans la régulation physiologique de CEBPA dans l'hématopoïèse. La présence initiale de CEBPA est essentielle à la transformation par de nombreuses oncoprotéines. En revanche, l'expression de CEBPA n'est plus nécessaire après la transformation. Cela suggère que CEBPA permet d'atteindre un stade de différenciation permissif pour l'activité transformante d'oncoprotéines comme les fusions MLL. En parallèle à ces effets instructifs de la différenciation, des travaux récents ont mis en évidence un rôle inattendu de CEBPA dans les cellules souches. Paradoxalement, dans ce contexte, CEBPA est responsable du maintien de l'arrêt de cycle. Son excision induit une hyperprolifération avec accumulation de progéniteurs, suivie d'un épuisement des souches et d'une aplasie médullaire. Ainsi, comme dans le cas de Runx1, l'activité de ce facteur dépend de manière critique du stade exact de différenciation de la cellule cible.

Paradoxalement, l'expression d'un mutant de liaison à l'ADN induit des états préleucémiques, ce que l'excision complète du gène ne fait jamais. Cela suggère, comme dans le cas de Runx1, qu'il existe des signalisations essentielles qui ne passent pas par la fixation sur les sites canoniques. Ce gène à exon unique peut être traduit à partir de deux codons ATG différents. L'initiation par le site interne permet l'expression d'une protéine (p30) qui n'interagit pas avec E2F et ne contrôle pas le cycle cellulaire. La surexpression de cette isoforme a des propriétés oncogéniques in vivo. CEBPA est muté dans un grand nombre de leucémies myéloïdes. Les leucémies avec mutations bi-alléliques de CEBPA combinent une haplo-insuffisance de CEBPA (un événement pro-oncogénique par lui-même), avec des codons-stop situés entre les deux codons ATG, forçant ainsi l'expression de p30. Outre les mutations, l'expression de CEBPA est indirectement ciblée par de très nombreux autres 
mécanismes oncogéniques : plusieurs protéines de fusions répriment l'expression de son gène, d'autres se fixent à son ARNm, enfin, de nombreux oncogènes interagissent directement avec la protéine. Tous ces mécanismes interfèrent avec les fonctions de ce régulateur maître. Enfin, comme pour Runx1, il existe des mutations germinales de CEBPA prédisposant aux leucémies. Ces mutations sont le plus souvent situées entre les deux codons ATG, facilitant l'expression de p30. La mutation ultérieure de l'allèle normal conduit au développement de leucémies.

L'ensemble de ces observations montre bien le rôle clé de ces facteurs maitres de différenciation, mais souligne aussi la complexité des relations entre celle-ci et l'autorenouvellement, en particulier pour les formes mutées ou fusionnées de ces facteurs. Pourtant, la compréhension de ces mécanismes est un préalable à une intervention pharmacologique sur ces voies maîtresses.

\section{COLLOQUE - MASTER TRANSCRIPTIONAL REGULATORS AND CANCER}

Les invités à ce colloque (Bruno Amati, Olivier Delattre, Nicholas Hastie, Ariel Melnick, H.R. Delwel, Claus Nerlov, Georges Lacaud, Corinne Bertolotto) ont exposé leurs travaux les plus récents sur des problématiques diverses. Les aspects technologiques, développementaux, médicaux et thérapeutiques ont été présentés.

\section{COURS À L'EXTÉRIEUR - RÉGULATION DE L'EXPRESSION GÉNÉTIQUE ET TRANSFORMATION CELLULAIRE}

Ces deux cours, destinés aux étudiants de la filière francophone de l'école de médecine de l'université JiaoTong de Shanghai, ont eu lieu au mois d'avril 2018. Ils permettent de maintenir la tradition francophone dans l'un des plus prestigieux hôpitaux de Chine et d'entretenir les liens historiques et scientifiques très forts. Dans la suite de l'enseignement dispensé à Paris, ces deux cours ont présenté des notions sur la régulation de l'expression génétique, en prenant comme modèle P53, les récepteurs nucléaires, Runx1 ou CEBPA.

\section{RECHERCHE}

La leucémie aiguë promyélocytaire (LAP) est déclenchée par une translocation chromosomique qui conduit à la formation d'une protéine de fusion PML/RARA, nécessaire et suffisante pour la transformation leucémique. PML/RARA est une protéine multifonctions qui exerce des effets dominants négatifs aussi bien sur le contrôle de l'expression génétique par RARA que sur la biogénèse des corps nucléaires PML. L'existence de deux agents thérapeutiques, l'acide rétinoïque et l'arsenic, fait l'élégance et la puissance de ce système. L'un et l'autre de ces médicaments se fixent directement à PML/RARA, changeant profondément ses fonctions biologiques. L'acide rétinoïque transforme PML/RARA en un activateur de l'expression génétique. L'arsenic induit l'agrégation de PML et PML/RARA, permettant ainsi la reformation des corps nucléaires PML. Enfin, ces deux agents induisent la destruction de PML/RARA par le protéasome. Utilisant des approches pharmacologiques et génétiques, nous avons démontré que cette dégradation de 
PML/RARA est responsable de l'éradication de la maladie par la combinaison acide rétinoïque/arsenic. Celle-ci nécessite la présence du gène PML normal requis pour l'activation de P53, un gène clé de la sénescence. L'analyse de patients historiques résistant au traitement a mis en évidence des mutations de l'allèle normal de PML, démontrant l'implication de PML dans la réponse.

L'équipe a beaucoup investi dans l'étude des fonctions des corps nucléaire PML in vivo. Cette année a vu l'aboutissement de travaux collaboratifs menés avec l'équipe de G. Meng à Shanghai. Nous avons démontré que la tétramérisation du domaine à doigt de zinc de type RING est indispensable à la formation des corps nucléaires PML et à la conjugaison ultérieure par SUMO. Nous avons également démontré que PML joue un rôle critique dans le contrôle du potentiel redox in vivo à travers la modulation de l'activation de P53 et de ses cibles anti-oxydantes. Ces travaux apportent des éclairages nouveaux sur la biologie cellulaire des corps nucléaires PML, comme sur leur rôle physiologique.

Nous avons mené une étude de génomique très fine sur un groupe exceptionnel de patients leucémiques ayant rechuté avec le traitement classique acide rétinoïque/ chimiothérapie. Un premier groupe de patients présente dès le diagnostic des activations d'oncogènes majeures, en plus de PML/RARA. Ces rechutes sont directement issues du clone leucémique initial et présentent souvent d'autres anomalies pro-transformantes. Nous proposons que le traitement ne parvienne pas à induire la sénescence des blastes leucémiques, expliquant la rechute. En plus de cette situation attendue, nous avons identifié une autre classe de rechute, souvent associée à la présence initiale de mutation de FLT3. Dans ces cas, les signatures moléculaires des rechutes (les mutations non associées à la transformation) sont totalement distinctes de celles qui sont présentes au diagnostic. Cette situation inattendue implique l'existence - et la persistance longtemps après traitement - d'un clone préleucémique non proliférant n'exprimant que PML/RARA, insensible à l'acide rétinoïque, mais capable de réinitier la leucémie jusqu'à 13 ans après le diagnostic.

D'autres travaux - qui viennent d'être soutenus par un contrat de l'ERC cherchent à comprendre les mécanismes moléculaires impliqués dans la sénescence PML-dépendante et à identifier des situations pathologiques où, comme dans la LAP, l'activation des corps PML pourrait avoir un bénéfice thérapeutique.

\section{Publications}

Wang P., Benhenda S., Wu H., Lallemand-Breitenbach V., Zhen T., Jollivet F., Peres L., Li Y., Chen S.-J., Chen Z., DE ThÉ H. et MENG G. «RING tetramerization is required for nuclear body biogenesis and PML sumoylation », Nature Communications, vol. 9, no 1, 2018, p. 1277, DOI : 10.1038/s41467-018-03498-0.

Wang L., Liu X., Wang H., Yuan H., Chen S., Chen Z., De Thé H., Zhou J. et Zhu J., «RNF4 regulates zebrafish granulopoiesis through the DNMT1-C/EBP $\alpha$ axis », FASEB journal, vol. 32, no 9, 2018, p. 4930-4940, DOI : 10.1096/fj.201701450RR.

Lehmann-Che J., Bally C., Letouzé E., Berthier C., Yuan H., Jollivet F., Ades L., Cassinat B., Hirsch P., Pigneux A., Mozziconacci M.-J., Kogan S., Fenaux P. et de THÉ H., «Dual origin of relapses in retinoic-acid resistant acute promyelocytic leukemia », Nature Communications, vol. 9, no 1, 2018, p. 2047, DOI : 10.1038/s41467-018-04384-5.

LALlEMAND-BReITENBACH V. et DE THÉ H., «PML nuclear bodies: from architecture to function », Current Opinion in Cell Biology, vol. 52, 2018, p. 154-161, DOI : 10.1016/j. ceb.2018.03.011. 
Gaillard C., Surianarayanan S., Bentley T., Warr M.R., Fitch B., Geng H., PAssegué E., DE THÉ H. et KogAn S.C., « Identification of IRF8 as a potent tumor suppressor in murine acute promyelocytic leukemia », Blood Advances, vol. 2, no 19, 2018, p. 2462-2466, DOI : 10.1182/bloodadvances.2018018929.

Dubuisson L., Lormières F., Fochi S., Turpin J., PAsquier A., Douceron E., Oliva A., Bazarbachi A., Lallemand-Breitenbach V., De Thé H., Journo C. et MahieuX R., «Stability of HTLV-2 antisense protein is controlled by PML nuclear bodies in a SUMOdependent manner », Oncogene, vol. 37, no 21, 2018, p. 2806-2816, DOI : 10.1038/s41388018-0163-X.

Di Costanzo A., Del Gaudio N., Conte L., Dell'Aversana C., Vermeulen M., de Thé H., Migliaccio A., Nebbioso A. et Altucci L., « The HDAC inhibitor SAHA regulates CBX2 stability via a SUMO-triggered ubiquitin-mediated pathway in leukemia », Oncogene, vol. 37, no 19, 2018, p. 2559-2572, DOI : 10.1038/s41388-018-0143-1.

DE THÉ H., « Differentiation therapy revisited », Nature Reviews. Cancer, vol. 18, n 2, 2018, p. 117-127, DOI : $10.1038 /$ nrc.2017.103. 